\title{
Compressive stress-electrical conductivity characteristics of multiwall carbon nanotube networks
}

\author{
P. Slobodian ${ }^{1}$ P. Riha ${ }^{2}$, A. Lengalova ${ }^{1}$ - P. Saha ${ }^{1}$
}

\begin{abstract}
A network of entangled multiwall carbon nanotubes is presented as a conductor whose conductivity is sensitive to compressive stress both in the course of monotonic stress growth and when loading/unloading cycles are imposed. The testing has shown as much as $100 \%$ network conductivity increase at the maximum applied stress. It indicates favorable properties of multiwall carbon nanotube networks for their use as stress-electric signal transducers. To model the conductivity-stress dependence, it is hypothesized that compression increases local contact forces between nanotubes, which results in more conductive contacts. The lack of detailed knowledge of the mechanism as well as an unclear shift from individual contacts to the whole network conductance behavior is circumvented with a statistical approach. In this respect, good data representation is reached using Weibull distribution for the description of distribution of nanotube contact resistance.
\end{abstract}

\section{Introduction}

Recent technological progress heavily relies on the use of materials that can offer advanced structural and functional capabilities. In this respect, entangled carbon nanotube (CNT) network structures show a great potential for developing highperformance polymer composites and enhanced sensors. CNT networks can proportionally transfer their unique properties into reinforced composite materials and films for sensors and bring substantial improvements in structural strength, electrical and thermal conductivity, electromagnetic interference shielding and other properties [1,2].

The first carbon nanotube network was fabricated by Walters et al. [3], The authors dispersed nanotubes into a liquid suspension and then filtered through fine filtration mesh. Consequently, pure nanotubes stuck to one another and formed a thin freestanding entangled structure, later dubbed buckypaper.

A recent study investigated the mechanical behavior of entangled mats of 
carbon nanotubes and several other fibers during compression and cyclic tests [4], The obtained hysteresis loop between loading and unloading was linked with mat morphology and motion, friction and rearrangement of fibers during compaction. However, the electric resistance of CNT networks at compression has only been measured in [5,6] and the electric properties of CNT particulate reinforcement of polymers, for instance, in [7,8], The obtained data were analyzed to get an estimate of the resistance of CNT tangles and the contact resistance between nanotubes [5]. The aim to reveal the effect of surfacedoping of MWCNT networks on their pressure sensitivity is pursued in [6].

Though the papers $[5,6]$ bring basic information on CNT pressure sensing, the aim of this paper is more complex: to study the electrical conductivity of multiwall carbon nanotube (MWCNT) both in the course of monotonic stress growth and when loading/unloading cycles are imposed. Our MWCNT networks, prepared by filtration to the thickness of several hundred micrometers, show a potential for sensor applications.

\section{Experimental}

Purified MWCNT produced by acetylene CVD method were supplied by Sun Nanotech Co. Ltd., China. According to the supplier, the nanotube diameter is $10-30 \mathrm{~nm}$, length 1-10 $\mathrm{am}$, purity $>90 \%$ and (volume) resistivity $0.12 \mathrm{Qcm}$. The nanotubes were used for the preparation of aqueous paste: $1.6 \mathrm{~g}$ of MWCNT and $\sim 50 \mathrm{ml}$ of deionized water were mixed with the help of a mortar and pestle. The paste was then diluted in deionized water with sodium dodecyl sulfate (SDS) and 1-pentanol. Consequently, $\mathrm{NaOH}$ aqueous solution was added to adjust $\mathrm{pH}$ to the value of 10 [9]. The final nanotube concentration in the suspension was $0.3 \mathrm{wt} . \%$, concentrations of SDS and 1-pentanol $0.1 \mathrm{M}$ and $0.14 \mathrm{M}$, respectively. The suspension was sonicated in Dr. Hielscher GmbH apparatus (ultrasonic horn S7, amplitude 88 (am, power density $300 \mathrm{~W} / \mathrm{cm}$, frequency 24 $\mathrm{kHz}$ ) for 2 hours and the temperature of ca $50^{\circ} \mathrm{C}$.

For making an entangled MWCNT network on a polyurethane porous membrane [10], a vacuum filtration method was used. The formed disk-shaped network was washed several times by deionized water and methanol in situ, then peeled off from the membrane and dried between filtering papers. The thickness of the obtained disks (Fig. la) was proportional to volume of filtrated dispersion (filtrating of $1.64 \mathrm{ml}$ of dispersion per $1 \mathrm{~cm}^{2}$ of filter area yields a network thickness 100 jj.m). The thickness of network samples used for stress-conductivity tests was in the range of $400-450 \mathrm{jj}, \mathrm{m}$.

The structure of MWCNT network was investigated with a scanning electron microscope (SEM) made by Vega LMU (Tescan s.r.o., Czech Republic). The sample taken from the disk was first deposited onto carbon targets and covered with a thin $\mathrm{Au} / \mathrm{Pd}$ layer. Observations were performed using secondary electrons.

Pure MWCNT were also analyzed via transmission electron microscopy (TEM) using microscope JEOL JEM 2010 at the accelerating voltage of $160 \mathrm{kV}$. The sample for TEM was prepared on 300 mesh copper grid with a carbon film (SPI, USA) from MWCNT dispersion in acetone, which was prepared by ultrasonication, deposited on the grid and dried.

The network was tested for deformation using a simple set-up. The network stripe (length $10 \mathrm{~mm}$ and width $8 \mathrm{~mm}$ ) cut out from the manufactured disks of 
entangled CNT was first stepwise compressed between two glasses to the maximum value with a $60 \mathrm{~s}$ delay of strain reading in each step. Then the down- stress curve was measured in the same manner.

The conductivity characteristic of network stripes with dimensions given above was measured in a similar way as deformation. The loading area between glass plates was $8 \times 8$ $\mathrm{mm}$ and the deformation resolution better than $3 \%$. Two electrical contacts were fixed to the stripe by silver colloid electro-conductive paint Dotite D-550 (SPI Supplies) and the electrical conductivity was measured lengthwise by the two-point technique using multimeter Sefram 7338. The multimeter was calibrated before each measurement by a standardized ceramic resistor.

\section{Results and discussion}

\section{Experimental results}

The structure of the upper surface of entangled nanotubes can be seen in Fig. lb as SEM micrograph. After filtration of MWCNT suspension the network was dried, which caused its shrinkage by about $7 \%$. The porosity was calculated from relation $\langle/\rangle=\backslash$ $p_{\text {net }} / p_{M W C N T}$, where $p_{\text {net }}=0.56 \pm 0.03 \mathrm{~g} / \mathrm{cm}^{3}$ denotes the measured apparent density of the nanotube network. The values correspond to those given in literature; for instance, $p_{\text {net }}=$ $0.54 \mathrm{~g} / \mathrm{cm}^{3}$ for MWCNT network [11]

or very similar value for SWCNT network $p_{n e t}=0.46-0.55 \mathrm{~g} / \mathrm{cm}^{3}$ [12]. The measured average density of nanotubes $p_{M W C N T}=\backslash . l \mathrm{~g} / \mathrm{cm}^{3}$ is very close to the theoretical value, i.e. $1.8 \mathrm{~g} / \mathrm{cm}[13]$.

To find out the length, thickness, number of wall layers and defects of nanotubes, TEM analysis was used. Its results slightly differ from the properties declared by the manufacturer. A representative sample of nanotubes deposited on copper grid with carbon film is shown in Fig. 2a. From the micrograph the diameter of individual nanotubes was determined to be between 10 and $60 \underline{\mathrm{nm}}$ (100 measurements) with the average diameter and standard deviation of $15 \pm 6 \mathrm{~nm}$, their length from tenths of micron up to 3 |_im. The maximum aspect ratio of the measured nanotubes is thus about 300 . The structure of an individual nanotube is demonstrated in Fig. 2b. As can be seen, it consists of about 15 rolled layers of graphene, with the interlayer distance of ca $0.35 \mathrm{~nm}$. The outer layer seems to be wrinkled, probably due to different functional groups created by oxidation when MWCNT are exposed to environment after tube production.

Mechanical properties of manufactured structures were followed in the course of twelve compression and relaxation cycles with cyclic accumulation of residual strain. The results in the form of compressive stress vs. strain dependence are presented in Fig. 3. The accumulation of residual strain is often called ratcheting and the minimum strain in each cycle is defined as ratcheting strain, $s_{r}$. In MWCNT network ratcheting strain appears after the first compression cycle, most probably due to the initial deformation of porous structure and blocked reverse motion of nanotubes inside the compact network, as hypothesized in [5]. Thus the ability of the network to be repeatedly highly compressed is reduced. Moreover, during successive cycles of loading and unloading the change of ratcheting strain 
per cycle decreases and tends to reach a constant value of $s_{\mathrm{r}}$, as follows from Fig. 4.

The pivotal property investigated in this study was electrical conductivity of a MWCNT network constituting a complex serial-parallel circuit. The measured data are shown in Fig. 5 as a plot of conductivity values $a$ vs. applied compressive stress $r$. Compression causes a conductivity change during both the up-stress and down-stress periods due to specific deformation of porous structure. According to [5], the local contact forces increase during compression, allowing a better contact of nanotubes, which in turn leads to the decrease of contact resistance between crossing nanotubes; in release the dependence is just the opposite. At the same time, the possible effect of the distance between contacts on CNT tangle resistance is considered in [5]. The distance between contacts may decrease during compression owing to evoked relative motion of nanotubes, which corresponds to a lower intrinsic resistance of nanotube segments between contacts. Last but not least, compression may also bend the nanotubes sideways, which results in more contacts between nanotubes [14]. Since the contact points may act as parallel resistors, their increasing number causes an enhancement of MWCNT network conductivity.

The conductance mechanisms are apparently not reversible in the initial cycle since the down-stress curve indicates residual conductivity increase in the off-load state. Nevertheless, the ongoing compression cycles have a stabilizing effect on the conductivity-compression loops similarly to their effect on mechanical properties. The conductivity enhancement $\mathrm{cr}$,, defined as the residual minimum conductivity during each cycle, increases with the increasing number of cycles, and tends to reach a constant value, Fig. 6.

\section{Stochastic model of MWCNT network conductivity}

The conductivity of a MWCNT network subjected to compressive stress is considered to be governed by the aforementioned mechanisms ranging from the contact resistance between nanotubes through nanotube conduction to the network architecture. The complexity of network ability to conduct an electric current limits the application of classical electricity concepts for finding a relation between local contacts and the macroscopic network response to compressive strain. For this reason, we translate the results of experimental observation into a probabilistic scheme. Within the framework of this scheme, we assume that the distribution of local conductive points enables to describe the joint probability for the total network conductivity change under compressive stress $t$ by the cumulative distribution or unreliability function $F(T)$ for the two-parameter Weibull distribution

$$
\operatorname{Pr}(\tau)=F(\tau)=1-\exp \left[-\left(\tau / \tau_{0}\right)^{m}\right]
$$

where $F(t)$ is an increasing function, $0<F(T)<1$, and describes the probability of network conductivity change $\operatorname{Pr}(\mathrm{r})$ under the stress no greater than $T$. The two Weibull parameters are the shape parameter $m$ and the scale parameter $r_{0}$.

The assumption that the probability of the whole network conductivity follows 
the Weibull distribution is substantiated in Fig. 7. The figure shows that all the experimental points (for instance, the data for the loading period of the first cycle in Fig. $5)$ very closely follow a straight line when plotted in Weibull coordinates $\ln r \mathrm{vs} . \ln (\ln (1 /(1$ - $\left.\left.P_{1}\right)\right)$ ), where $P_{j}=(\mathrm{z}-0.5) / n$ and $i$ ranges from 1 to $\mathrm{n}$, which is

the number of tests. The goodness-of-fit to the straight line is reflected in the value of the correlation coefficient $r=0.99$.

The tendency of the measured increase of the macroscopic, i.e. network conductivity with compressive stress is bound to the probability of network conductivity change $\operatorname{Pr}(\mathrm{r})$ under stress no greater than $z$. Consequently, the following relation of the network conductivity $\langle j(t)$ to function $F(t)$ links appropriately the model prediction with the observed stress-dependent network conductivity increase

$$
\sigma(\tau)=\alpha+\beta\left(1-\exp \left[-\left(\tau / \tau_{0}\right)^{m}\right]\right)
$$

where $a$ and /? are location parameters. The reasonably good description of the measured data by the predictive relation (2), shown in Fig. 5 (solid and dotted lines) justifies the probability model chosen here for the MWCNT network conductivity change under compression.

\section{Final remarks}

The conductivity-compression characteristics for entangled CNT network structures of buckypaper produced by filtering a nanotube suspension have not been studied in details so far. Thus the primary aim of our research was to find out the effect of compression on network electrical conductivity when simple and repeated loading is exerted. The measurements have shown over $100 \%$ conductivity increase at the maximum applied compressive stress. It indicates a good potentiality of MWCNT entangled network to be applied as a compression sensing element. The conversion of compressive stress into improved conductivity is achieved by deformation of porous structure. The structure recovering mechanism projects into the ratcheting strain, the change of which decreases with the increasing number of applied cycles, and finally an asymptotic value of this residual strain is reached. During successive cycles of loading and unloading the nanotubes rearrangement becomes steady and MWCNT network reaches a stable stressstrain hysteresis loop shape. This mechanical stabilization is reflected also in conductivity data. The conductivity-stress loop is stable after the same number of cycles as in the mechanical cyclic loading. It shows that the entangled carbon nanotube network structure of buckypaper can be used as a sensing element of compressive stress, especially when the network is suitably deformed in advance.

Acknowledgements This project was financially supported by the Ministry of Education, Youth and Sports of the Czech Republic (MSM 7088352101), the Grant Agency of the Academy of Sciences of the Czech Republic (GAAV IAA200600803) and by the Fund of the Institute of Hydrodynamics AV0Z20600510. 


\section{References}

1. Thostenson ET, Li CY, Chou TW (2005) Compos Sei Technol 65: 491-516

2. Cao Q, Rogers JA (2009) Adv Mater 21: 29-53

3. Walters DA, Casavant MJ, Quin XC, Huffman CB, Boul PJ, Ericson LM, Haroz EH, O'Connel MJ, Smith K, Colbert DT, Smalley RE (2001) Chem Phys Lett 338:14-20

4. Poquillon D, Viguier B, Andrieu E (2005) J Mat Sei 40: 5963-5970

5. Allaoui A, Hoa SV, Evesque P, Bai J (2009) Scripta Mater 6: 628-631

6. Kukowecz A, Smajda R, Oze M, Schaefer B, Haspel H, Konya Z, Kiricsi I (2008) Phys Stat Sol 245:2331-2334

7. $\quad$ Simsek Y, Ozyuzer L, Seyham AT, Tanoglu M, Schulte K (2007) J Mater Sei 42:96899695

8. Chang L, Friedrich K, Ye L, Toro P (2009) J Mater Sei 44:4003-4012

9 Ham HT, Choi YS, Chung IJ (2005) Colloid Interf Sei 286:216-223

10. Kimmer D, Slobodian P, Petras D, Zatloukal M, Olejnik R, Saha P (2009) J Appl Polym Sei 111: 2711-2714

11. Wang D, Song PC, Liu CH, Wu W, Fan SS (2008) NANOTECHNOLOGY : 19(7) 1-6

12. Ashrafi B, Guan JW, Mirjalili V, Hubert P, Simard B, Johnston A (2010) COMPOS PART A-APPL S 41(9) 1184-1191

13. Xie XL, Mai YW, Zhou XP (2005) Mater Sei Eng 49: 89-112

14. Yaglioglu O, Hart AJ, Martens R, Slocum AH (2006) Rev Sei Instrum 77: 095105/1-3. 


\section{Figure captions}

Fig. 1 Free-standing randomly entangled MWCNT network (disk diameter 75 $\mathrm{mm}$, thickness $0.15 \mathrm{~mm}$ ), part a. SEM image of the surface and the cross-section of entangled MWCNT network of buckypaper, part b (scale $100 \mathrm{~nm}$ ), part c (scale $2^{\wedge} \mathrm{m}$ ), respectively.

Fig. 2 TEM image of MWCNT, part a (scale $500 \mathrm{~nm}$ ) and TEM detailed view of nanotube structure, part b (scale $10 \mathrm{~nm}$ )

Fig. 3 The loops of absolute stress-strain values for a compression test of MWCNT network subjected to 12 compression/expansion cycles (the network thickness $420^{\wedge} \cdot \mathrm{m}$ ). The solid lines (first loading and unloading cycle) and dotted lines (twelfth cycle) represent the power law fitting

Fig. 4 The absolute value of residual strain versus number of compression/expansion cycles. Data presented as a mean \pm standard deviation, $n=3$.

Fig. 5 Electrical conductivity-absolute value of compressive stress loops for MWCNT network subjected to 12 successive compression/expansion cycles (network thickness 420 |_im). ). The solid lines (first loading and unloading cycle) and dotted lines (twelfth cycle) represent the prediction given by Equation (2)

Fig. 6 Conductivity enhancement in each cycle vs. number of compression/expansion cycles. Data presented as a mean \pm standard deviation, $n=3$.

Fig. 7 Weibull plot for compressive strain (absolute value) deforming conductive MWCNT network 
Fig. 1
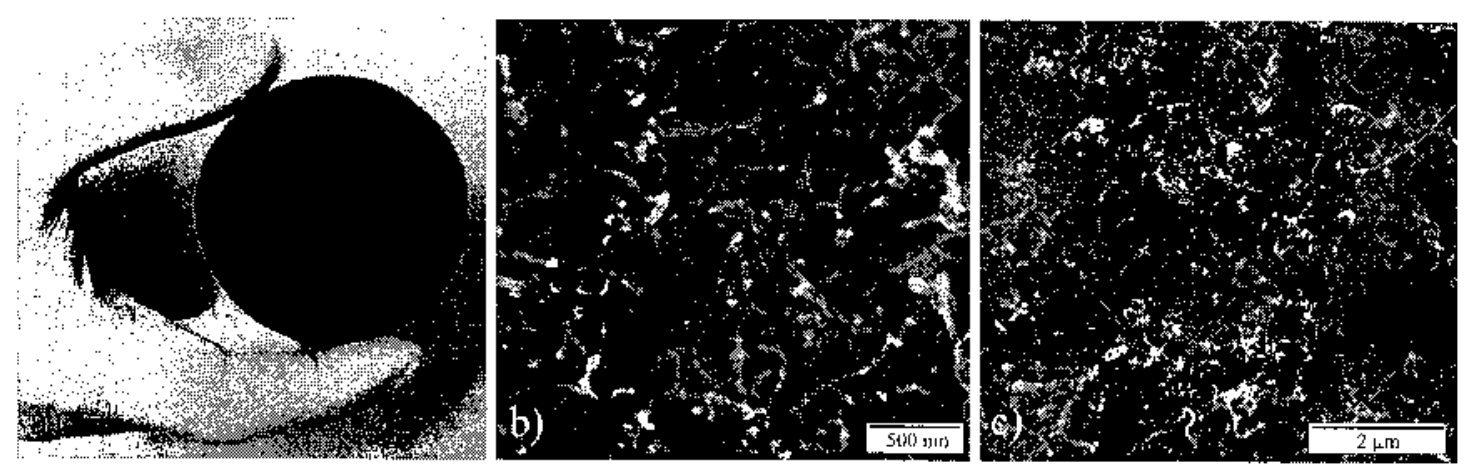

Fig. 2

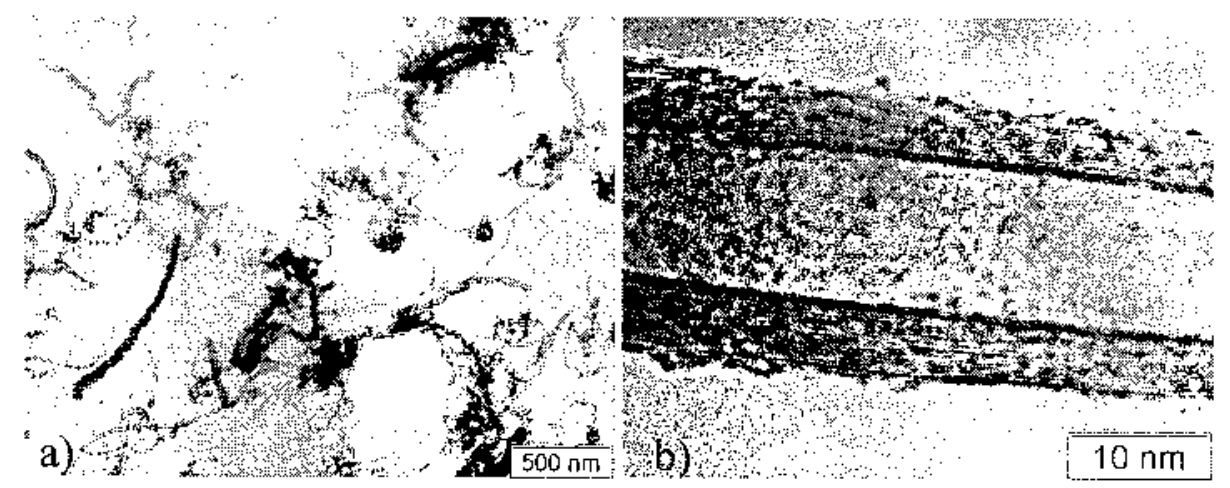

Fig. 3

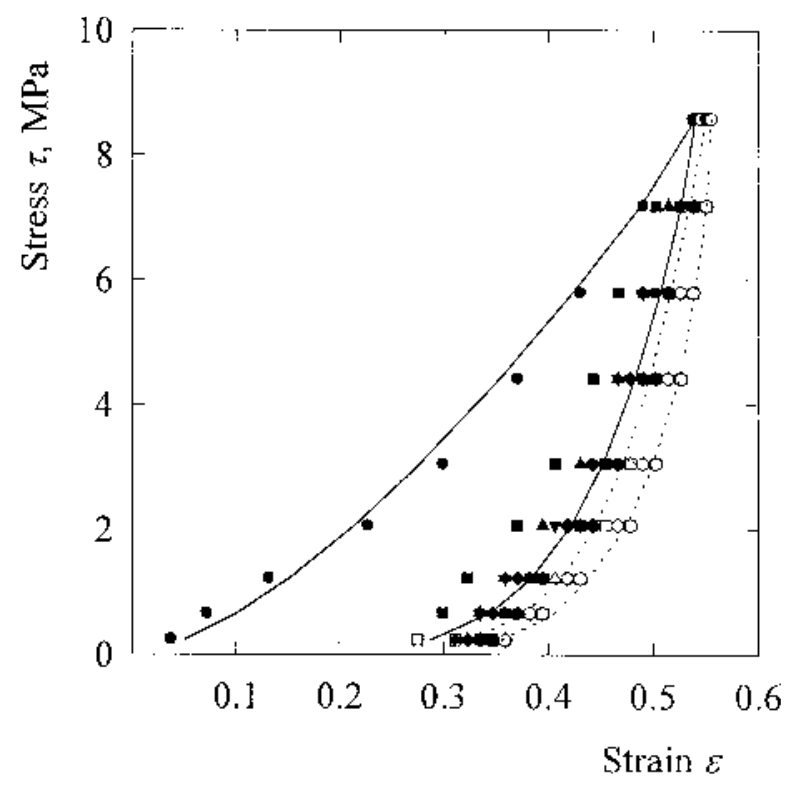


Fig. 5

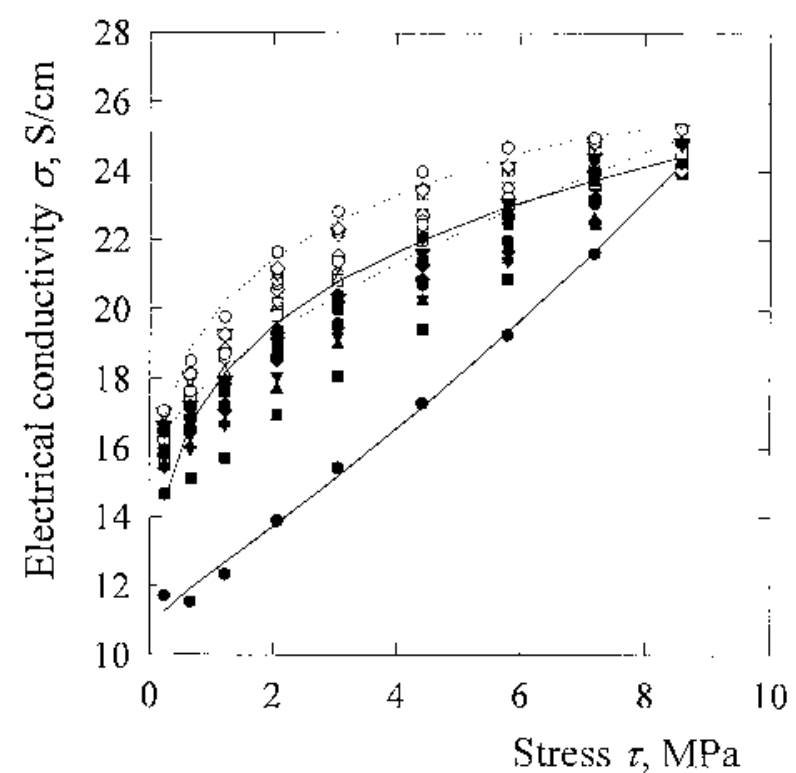


Fig. 4

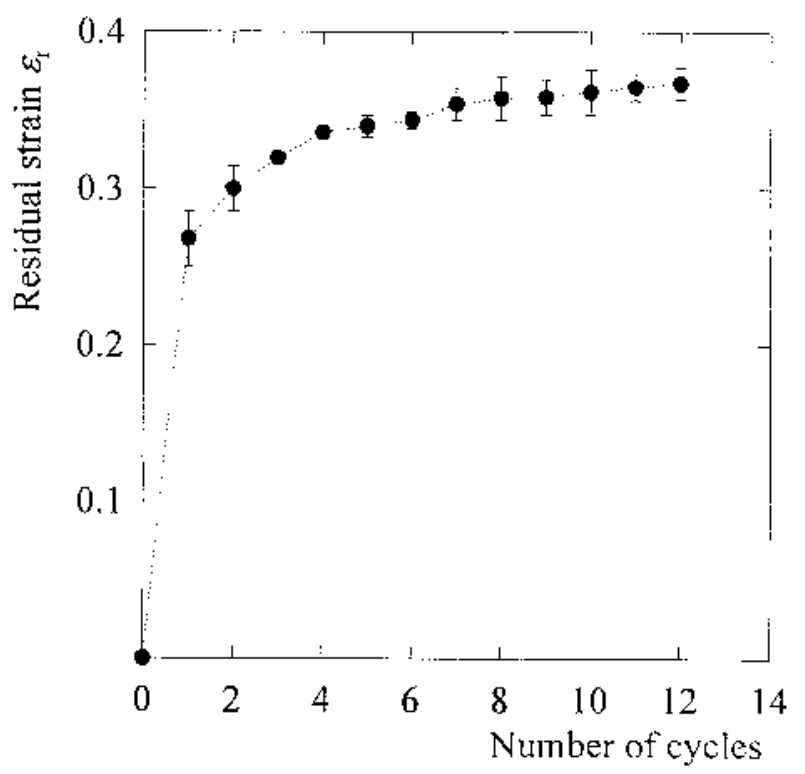


Fig. 6

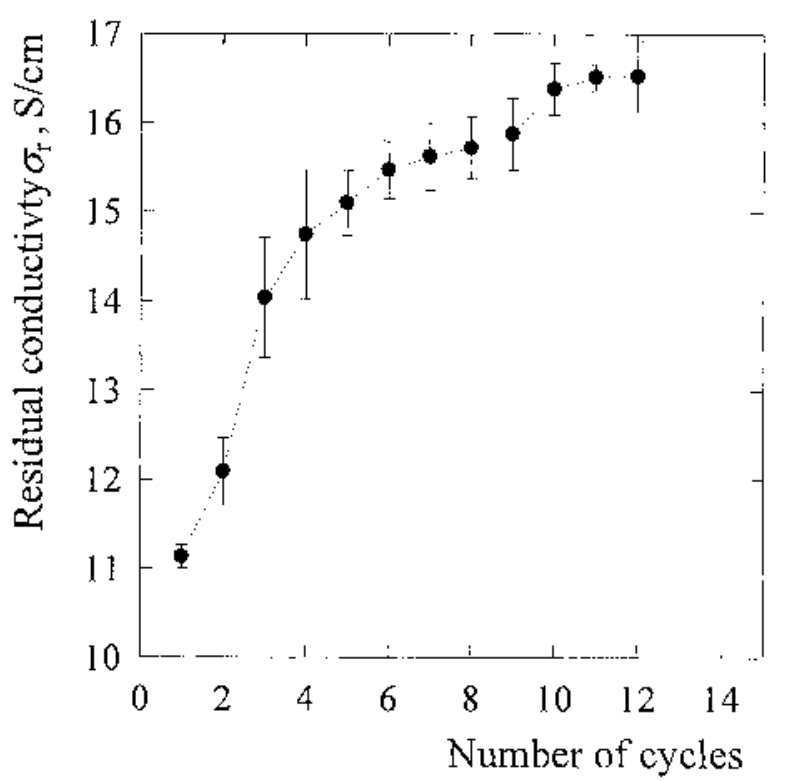

Fig. 7

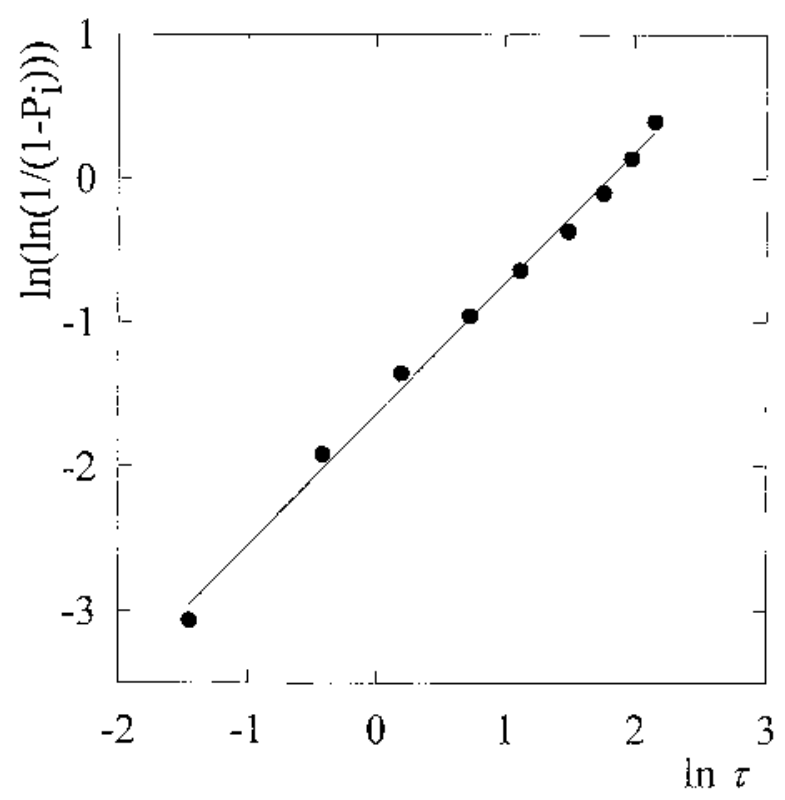

